\title{
CIRCULATING SERUM ADIPONECTIN AND OXIDATIVE STRESS BIOMARKERS IN PREDIABETES AND TYPE 2 DIABETES MELLITUS PATIENTS
}

\author{
RACHNA SHARMA*, SATYANARAYANA P, PALLAVI ANAND, SHRAWAN KUMAR \\ Department of Biochemistry, Rama Medical College, Hospital and Research Centre, Kanpur, Uttar Pradesh, India. \\ Email: drrachna99@gmail.com
}

Received: 30 July 2019, Revised and Accepted: 10 October 2019

\section{ABSTRACT}

Aims and Objectives: Adipocytokines and oxidative stress have been independently studied in various morbidities, but their interrelationship in mediating insulin resistance and type 2 diabetes mellitus is still unclear. Thus, the present study was aimed at evaluating malondialdehyde (MDA) levels in pre-diabetes and diabetics and correlating it with adiponectin levels.

Methods: Fifty controls, 50 pre-diabetic, and 50 diabetic patients were enrolled. Plasma MDA, uric acid, and adiponectin were measured in the blood samples. Serum MDA adiponectin was analyzed by enzyme-linked immunosorbent assay and uric acid was analyzed by uricase method.

Results: Pre-diabetic and diabetic patients had higher levels of MDA and uric acid, while the level of adiponectin was low compared to controls. Correlation of MDA was positive with uric acid but negative with adiponectin in diabetic patients. Likewise, adiponectin and uric acid were also correlated negatively. In pre-diabetes, adiponectin was significantly and negatively correlated with MDA and uric acid.

Conclusions: Pre-diabetic and diabetic patients have increased oxidative stress, which is also linked with adipokine abnormalities. From this study, we observed that oxidative stress suppresses adiponectin production which is the protective adipokine in hyperglycemia. Thus, oxidative stress may serve as an indicator or target for in the control of hyperglycemic stress.

Keywords: Oxidative stress, Pre-diabetes, Diabetes, Malondialdehyde.

(C) 2019 The Authors. Published by Innovare Academic Sciences Pvt Ltd. This is an open access article under the CC BY license (http://creativecommons. org/licenses/by/4. 0/) DOI: http://dx.doi.org/10.22159/ajpcr.2019.v12i12.35142

\section{INTRODUCTION}

Both pre-diabetes and diabetes are a metabolic disorder induced by insulin resistance. According to the $\mathrm{WHO}$, about $9 \%$ of the population around the globe were affected with diabetes accounting for 1.5 million deaths in 2014 [1]. Another key factor responsible for insulin resistance is oxidative stress. It occurs due to an imbalance in free radical production and their detoxification by the anti-oxidant system of the body [2]. The potent free radicals are reactive oxygen species such as superoxide, hydroxyl ions, and hydrogen peroxide, which are produced in low levels in mitochondria and peroxisomes in normal physiological conditions and are sufficiently acted on by the anti-oxidant defense system [3,4]. However, if the oxidative stress becomes chronic, it causes damage to biomolecules such as DNA, proteins, and lipids resulting in the development of chronic diseases such as diabetes, cancer, and cardiovascular diseases. Chronic oxidative stress especially is more harmful to pancreatic $\beta$-cells as these cells express lower amount of anti-oxidant enzymes [5]. Researches have revealed a strong correlation of oxidative stress with the insulin resistance and the stages of diabetes. However, the knowledge regarding this phenomenon is incomplete. Oxidative stress is also implicated to have roles in alteration in adipokine release, but this area has been explored inadequately. Adipokine that is shown to have beneficial role in insulin resistance and oxidative stress is adiponectin. Although adiponectin functions to reduce both insulin resistance and oxidative damage, the exact mechanism of such association is not clear [6,7]. Furthermore, studies showing a link between oxidative stress and diabetes are available, but paucity lies with regards to pre-diabetes. With this background, we in this study aimed to explore the relationship between oxidative stress and adiponectin in pre-diabetic and diabetic patients.

\section{METHODS}

This study was conducted in Rama Medical College, Hospital and Research Centre Rama University, Kanpur, Uttar Pradesh, India. We included 50 controls, 50 pre-diabetic patients, and 50 diabetic patients. Structured proforma was prepared to record a detailed history of the patients and the study was initiated with the approval of ethical committee of the institute and written consent of the participants.

\section{Inclusion criteria}

Type 2 diabetic patients with age $\geq 30$ were selected. The criteria for diabetes mellitus were as follows:

- Fasting blood glucose (FBG): $\geq 126 \mathrm{mg} / \mathrm{dl}$

- Postprandial blood glucose: $\geq 200 \mathrm{mg} / \mathrm{dl}$ along with the cardinal symptoms.

\section{Exclusion criteria}

- Patients with age $<30$ were excluded from the study

- Patients with any illness such as type 1 diabetes mellitus, inflammatory states, malignancy, hypothyroidism, any complications of diabetes, or any hormonal derangement were excluded from the study.

The patients were asked to give their blood samples after overnight fasting and the $2 \mathrm{~h}$ after the carbohydrate-rich meal (postprandial sample). The anthropometric variables such as height and weight were recorded for each patient. In the blood samples collected, the parameters such as blood glucose, glycated hemoglobin (HbA1c), lipids, uric acid, malondialdehyde (MDA), and adiponectin were estimated by kit-based methods.

\section{Statistical analysis}

The data were maintained as mean \pm SD. Statistical analysis was carried out with SPSS 18 tool. The comparison of parameters in the study groups was done by student's t-test, while correlation was determined by Pearson's correlation coefficient. $\mathrm{p}<0.05$ indicated statistical significance. 
Table 1: Comparison of basic parameters

\begin{tabular}{|c|c|c|c|c|c|c|}
\hline Parameter & Control & Pre-diabetes & Diabetes & CvsP & CvsD & PvsD \\
\hline Age & $49.57 \pm 10.23$ & $50.01 \pm 9.84$ & $52.81 \pm 9.66$ & 0.0001 & 0.1067 & 0.1542 \\
\hline Body mass index & $23.72 \pm 3.55$ & $25.11 \pm 2.5$ & $27.17 \pm 3.14$ & 0.3138 & 0.0154 & 0.0005 \\
\hline Fasting blood glucose & $82.12 \pm 9.38$ & $120.97 \pm 3.73$ & $138.2 \pm 9.86$ & $0.0001^{* *}$ & $0.0001^{* *}$ & $0.0001^{* *}$ \\
\hline Postprandial blood glucose & $123.43 \pm 10.41$ & $159.24 \pm 7.11$ & $262.3 \pm 12.52$ & $0.0001^{* *}$ & $0.0001^{* *}$ & $0.0001^{* *}$ \\
\hline Glycated hemoglobin & $4.99 \pm 0.4$ & $5.94 \pm 0.21$ & $9.36 \pm 1.28$ & $0.0001^{* *}$ & $0.0001^{* *}$ & $0.0001^{* *}$ \\
\hline Cholesterol & $153.31 \pm 3.11$ & $172.23 \pm 8.35$ & $244.8 \pm 9.49$ & $0.0001^{* *}$ & $0.0001^{* *}$ & $0.0001^{* *}$ \\
\hline High-density lipoproteins & $83.33 \pm 7.89$ & $42.57 \pm 5$ & $39.85 \pm 3.41$ & $0.0001^{* *}$ & $0.0001^{* *}$ & $0.0001^{* *}$ \\
\hline Low-density lipoproteins & $42.03 \pm 5.11$ & $84.29 \pm 9$ & $88.58 \pm 7.99$ & $0.0001^{* *}$ & $0.0001^{* *}$ & $0.0001^{* *}$ \\
\hline
\end{tabular}

Table 2: Comparison of major parameters

\begin{tabular}{llllll}
\hline Parameter & Control & Pre-diabetes & Diabetes & CvsP & CvsD \\
\hline Adiponectin & $10.74 \pm 2.24$ & $9.48 \pm 2.41$ & $6.45 \pm 2.01$ & $0.0001^{* *}$ & 0.008 \\
Malondialdehyde & $2.98 \pm 1.59$ & $4.06 \pm 2.23$ & $9.14 \pm 2.68$ & 0.0064 & $0.0001^{* *}$ \\
Uric acid & $4.52 \pm 0.89$ & $6 \pm 0.77$ & $5.24 \pm 1.28$ & $0.0001^{* *}$ & $0.0001^{* *}$ \\
\hline
\end{tabular}

Table 3: Correlation between the parameters in pre-diabetes

\begin{tabular}{llllll}
\hline Parameter & FBG & HbA1c & Uric acid & Adiponectin & MDA \\
\hline FBG & - & 0.056 & -0.06 & 0.06 & 0.001 \\
HbA1C & 0.056 & - & $-0.15^{*}$ & -0.03 & -0.06 \\
Uric acid & -0.06 & $-0.15^{*}$ & - & $-0.16^{*}$ & -0.002 \\
Adiponectin & 0.06 & -0.03 & $-0.16^{*}$ & - & $-0.27^{* *}$ \\
MDA & 0.001 & -0.06 & -0.002 & $-0.27^{* *}$ & - \\
\hline
\end{tabular}

MDA: Malondialdehyde, HbA1C: Glycated hemoglobin, FBG: Fasting blood glucose

Table 4: Correlation between the parameters in diabetes

\begin{tabular}{llllll}
\hline Parameter & FBG & HbA1c & Uric acid & Adiponectin & MDA \\
\hline FBG & - & $0.6^{* *}$ & $0.19^{* *}$ & $-0.29^{* *}$ & $0.44^{* *}$ \\
HbA1C & $0.6^{* *}$ & - & $0.45^{* *}$ & $-0.43^{* *}$ & $0.61^{* *}$ \\
Uric acid & $0.19^{* *}$ & $0.45^{* *}$ & - & $-0.21^{* *}$ & $0.28^{* *}$ \\
Adiponectin & $-0.29^{* *}$ & $-0.43^{* *}$ & $-0.21^{* *}$ & - & $-0.59^{* *}$ \\
MDA & $0.44^{* *}$ & $0.61^{* *}$ & $0.28^{* *}$ & $-0.59^{* *}$ & - \\
\hline
\end{tabular}

MDA: Malondialdehyde, HbA1C: Glycated hemoglobin, FBG: Fasting blood glucose

\section{RESULTS}

The basic parameters included in this study are shown in Table 1. Age, body mass index, FBG, postprandial blood glucose, and HbA1C were significantly high in both pre-diabetic and diabetic patients compared to controls. Similar was the case with cholesterol, triglycerides, and low-density lipoproteins, while the level of high-density lipoproteins was significantly low.

In Table 2, we compared uric acid, adiponectin, and MDA. Both uric acid and MDA were significantly high and adiponectin was significantly low inpatient group compared to controls. Uric acid level was lower in diabetic patients compared to pre-diabetes.

In Table 3, adiponectin was significantly and negatively correlated with MDA and uric acid. Uric acid showed a significant inverse association with $\mathrm{HbA1C}$. In Table 4, we observed significant linear correlation of uric acid and MDA with FBG and $\mathrm{HbA1C}$ while it was inverse with adiponectin. Further adiponectin also showed significant and negative correlation FBG and $\mathrm{HbA1C}$, and the correlation was positive between MDA and uric acid.

\section{DISCUSSION}

Free radicals though are harmful do not cause damage to the body if they are produced normally under the normal physiological state as they are sufficiently scavenged by the anti-oxidants present in the body. However, if these reactive species are insufficiently scavenged, they start damaging cellular components especially lipids and DNA [8,9]. One of the well-studied markers of oxidative stress is MDA. It is the biomarker for lipid peroxidation and increases significantly in diabetes [10]. In this study, the level of MDA was significantly increased in prediabetes and diabetes compared to controls. Our result was in line with that of Noberasco et al. [11], Bhatia et al. [12], and Saad [13]. Similarly the level of adiponectin was decreased and uric acid was increased in the patients groups. According to Nakanishi et al. [14], adiponectin is associated inversely with the oxidative stress marker in diabetic patients. In accordance to this, we also observed negative association of adiponectin with MDA and uric acid. The association was statistically significant. The correlation between MDA and uric acid was linear.

Our findings support the implication that the derangement in glucose regulation can cause lipid peroxidation through protein glycation or insulin resistance induced generation of free radicals from metabolic pathways such as fatty acid oxidation and hexose monophosphate shunt. Hyperglycemia can also induce platelet aggregation that enhances prostaglandin and thromboxane production which are also the major sources of free radical [15]. MDA is also suggested to link protein glycation with lipid peroxidation and can induce inflammatory cytokines that act as trigger to further oxidative damage [16]. Excess production of free radicals further causes dysregulation in adipokine production as well as in the levels of interleukin-6, tumor necrosis factor- $\alpha$, plasminogen activator inhibitor, etc, leading to impaired glucose uptake in peripheral tissues and decreased production of insulin from pancreas [17]. According to previous reports, MDA promotes and adiponectin decreases oxidative stress in hyperglycemic patients.

\section{CONCLUSIONS}

Oxidative stress is a state of disbalance between free radical production and the anti-oxidant defense system. Hyperglycemia is the major contributor to oxidative stress through various metabolic pathways. MDA is a common biomarker of oxidative stress, while adiponectin has protective effect against oxidative stress. Thus, interventions to increase adiponectin and decrease MDA may produce beneficial outcomes in hyperglycemic patients. Since pre-diabetes is the latent state of hyperglycemia, timely management at this phase can prevent diabetes progression and complications of oxidative stress.

\section{ACKNOWLEDGMENT}

The first author thankfully acknowledge the management of Rama Medical College and Hospital, Kanpur, for supporting the study. 


\section{AUTHORS' CONTRIBUTION}

All authors listed above have made a substantial, direct, and intellectual contribution to the work and approved it for publication.

\section{CONFLICTS OF INTEREST}

The authors declare that they have no conflicts of interest.

\section{SOURCE OF FUNDING}

Self.

\section{REFERENCES}

1. Aguilar M, Bhuket T, Torres S, Liu B, Wong RJ. Prevalence of the metabolic syndrome in the United States, 2003-2012. JAMA 2015:313:1973-4.

2. Halliwell B. Antioxidants: The basics what they are and how to evaluate them. Adv Pharmacol 1997;38:3-20.

3. Schieber M, Chandel NS. ROS function in redox signaling and oxidative stress. Curr Biol 2014;24:R453-62.

4. Brand MD. Mitochondrial generation of superoxide and hydrogen peroxide as the source of mitochondrial redox signaling. Free Radic Biol Med 2016;100:14-31.

5. Sharma P, Kumar P, Sharma R, Prakash S. Assessment of cardiometabolic risk factors among local population of NCR. Asian J Pharm Clin Res 2017;10:212-3.

6. Kizer JR. A tangled threesome: Adiponectin, insulin sensitivity, and adiposity: Can mendelian randomization sort out causality? Diabetes 2013;62:1007-9.

7. Shehzad A, Iqbal W, Shehzad O, Lee YS. Adiponectin: Regulation of its production and its role in human diseases. Hormones (Athens) 2012;11:8-20.

8. Saad EA. Curative and protective effects of L-arginine on carbon tetrachloride-induced hepatotoxicity in mice. Biochem Biophys Res Commun 2012;423:147-51.

9. Saad EA, Habib SA. Effect of crude extracts of some medicinal plants on the osmotic stability of human erythrocytes in vitro. J Free Radic Antioxid Photon 2013;139:265-72.

10. Koch A, Sanson E, Voigt S, Helm A, Trautwein C, Tacke F, et al. Serum adiponectin upon admission to the intensive care unit may predict mortality in critically ill patients. J Crit Care 2011;26:166-74.

11. Noberasco G, Odetti P, Boeri D, Maiello M, Adezati L. Malondialdehyde (MDA) level in diabetic subjects. Relationship with blood glucose and glycosylated hemoglobin. Biomed Pharmacother 1991;45:193-6.

12. Bhatia S, Shukla R, Venkata Madhu S, Kaur Gambhir J, Madhava Prabhu K. Antioxidant status, lipid peroxidation and nitric oxide end products in patients of type 2 diabetes mellitus with nephropathy. Clin Biochem 2003;36:557-62.

13. Saad EA, Habib SA, Refai WA, Elfayoumy AA. Malondialdehyde, adiponectin, nitric oxide, C-reactive protein, tumor necrosis factoralpha and insulin resistance relationships and inter-relationships in Type 2 diabetes early stage. Is metformin alone adequate in this stage? Int J Pharm Pharm Sci 2017;9:176-81.

14. Nakanishi S, Yamane K, Kamei N, Nojima H, Okubo M, Kohno N. A protective effect of adiponectin against oxidative stress in Japanese Americans: The association between adiponectin or leptin and urinary isoprostane. Metabolism 2005;54:194-9.

15. David F, Farley J, Huang H, Lavoie JP, Laverty S. Cytokine and chemokine gene expression of IL-1beta stimulated equine articular chondrocytes. Vet Surg 2007;36:221-7.

16. Chokkalingam V, Tel J, Wimmers F, Liu X, Semenov S, Thiele J, et al. Probing cellular heterogeneity in cytokine-secreting immune cells using droplet-based microfluidics. Lab Chip 2013;13:4740-4.

17. Kocić R, Pavlović D, Kocić G, Pesić M. Susceptibility to oxidative stress, insulin resistance, and insulin secretory response in the development of diabetes from obesity. Vojnosanit Pregl $2007 ; 64: 391-7$. 